\title{
A Feature-based Method for MBD Model Exchange among Heterogeneous CAD Systems
}

\author{
Xie Weikang ${ }^{1,}$, , Zhao Gang ${ }^{1, b}$, Yu Yong ${ }^{1, c}$ and Wang Yaodong ${ }^{2, d}$ \\ ${ }^{1}$ School of Mechanical Engineering and Automation, Beijing University of Aeronautics and \\ Astronautics, Beijing 100191, China; \\ ${ }^{2}$ Institute of Telecommunication Satellite, China Academy of Space Technology, Beijing 100094, \\ China. \\ axieweikang2010@163.com, bzhaog@buaa.edu.cn, cyuyong@buaa.edu.cn, \\ dyaodong1217@163.com
}

Keywords: heterogeneous CAD; MBD model; feature-based; data exchange; non-geometric information.

\begin{abstract}
To solve the problem that missing feature and non-geometric information when MBD (Model Based Definition) model is exchanged among heterogeneous CAD systems, a feature-based heterogeneous MBD model exchange method is proposed considering geometric feature information, non-geometric information and the association between them. Firstly, use a neutral format to express the feature information and non-geometric information of MBD model and the association between them. Secondly, transform the geometric feature information and engineering note information at the same time and maintain the relationship between them through information extraction and reconstruction by using XML (Extensible Markup Language) file as neutral format. Finally, exchange a structural model of a certain type of aircraft between Pro/E and CATIA to verify the validity of the method.
\end{abstract}

\section{Introduction}

With the rapid development of economic globalization and digitalization technology, collaborative design for complex product by distributed in different locations, belong to different enterprises or departments has become a trend [1]. In the process of product collaborative design, due to reasons of function or economy, different enterprises or departments often choose various CAD systems. So, the study of data exchange method among heterogeneous CAD systems is extremely important for collaborative design. In the meantime, with the technological progress of manufacturing technology, large-scale equipment manufacture represented by aerospace is phasing in full three dimensional digitalization design and manufacturing methods of product based on MBD [2]. MBD model is widely adopted in collaborative design which is distinct from traditional CAD model. It has not only geometric information but also non-geometric information. Therefore, it needs to consider geometric and non-geometric information to exchange heterogeneous MBD model.

There are major two approaches to exchange data among heterogeneous CAD systems: geometry-based data exchange and feature-based data exchange [3]. Geometry-based data exchange is based on neutral data exchange standards (such as IGES, STEP). Yet the standards have remained largely restricted to the representation of final geometry, causing the design intent portrayed through construction history, features, parameters, and constraints to be discarded in the exchange process ${ }^{[3]}$. Feature-based data exchange can exchange high level semantic information (design features, parameters, and constraints) in the feature level. Hoffmann [4] firstly studied E-Rep (editable representation, E-Rep) to describe model through a series of modeling activities such as feature building, modifying and deleting, so as to transform the creating procedure of the model. Han ${ }^{[5-6]}$ et al. proposed a method to exchange data among heterogeneous CAD systems by concluding neural modeling command and using macro file (log file) as medium. Rappoport [7, 9] et al. proposed an UPR (Universal Product Representation) framework, summarized data type or modeling operation 
type supported by each CAD system, exchanged building history of model by feature rewrite mechanism. He $[10,11]$ et al. proposed a two-stage information retrieval and model reconstruction mechanism to ensure the integrity and accuracy of the data exchange through necessary human-computer interaction. The above researches mainly aimed at traditional CAD models, and they only considered geometrical information of CAD models. Because geometrical information and non-geometrical information are correlative in MBD model, so in view of the heterogeneous MBD model exchange, not only to preserve the design intent, should also exchange non-geometrical information associated with geometry information. Therefore, to exchange heterogeneous MBD model, this paper improve feature-based data exchange method. Firstly, use XML as unified format to express geometric feature information, non-geometrical information and their correlation. Then, exchange MBD model among heterogeneous CAD systems through information extraction and reconstruction. Finally, take a structural model of a certain type of aircraft as example to verify the validity of the method between Pro/E and CATIA.

\section{Heterogeneous MBD Model Exchange Method}

\subsection{Information Representation of Heterogeneous MBD Model}

MBD is an advanced digital definition method which attaches the information of all the relevant product design definition, process description, attributes and management in the product three-dimensional model [12]. Its core idea is to use an integrated three-dimensional entity model to express product definition information completely, using it as the only basic in the process of production. A full defined MBD dataset is shown in Fig.1 [13].

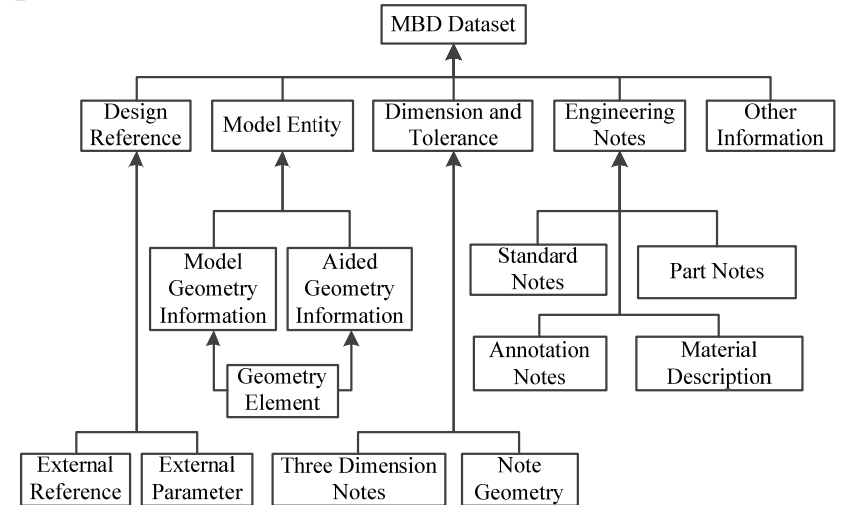

Fig.1 The structure of MBD dataset

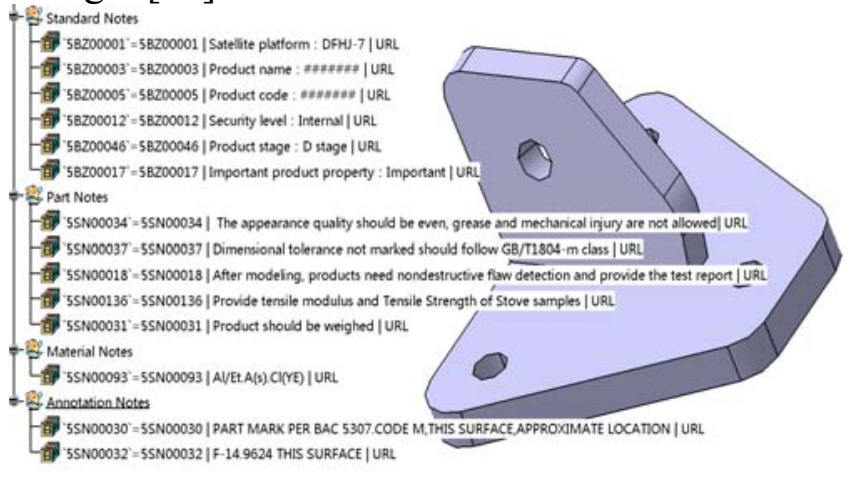

Fig.2 An instance of MBD dataset

MBD model is different from traditional three-dimensional CAD model. It has not only geometric feature information, but also 3D annotation information describing dimension and tolerance information of the model. In addition, it also contains engineering notes information including process, manufacturing and checking information during product development process. Fig. 2 shows a MBD model that belongs to MBD model history database of some astronautic enterprise.

Refer to the MBD dataset normative definition requirements put forward by Boeing Company [12], the MBD model information are divided into two types (Design Reference and Other Information is not considered in this paper): Geometric feature information and Engineering notes information. This paper use XML to represent the MBD model information.

\subsubsection{Geometric Feature Information Representation}

The geometric feature information of MBD model describes entity models' dimension and tolerance information. Through analyzing the features commonly used in the process of modeling, the features can be divided into three categories: sketch-based feature, dress-up feature and transformation feature.

(1) Sketch-based feature

Sketch-based feature is a kind of 3D entity feature based on 2D sketch, including extrude (add material/remove material), revolve (add material/remove material), hole and so on. 
Fig. 3 shows the information contained in sketch-based feature, including the feature type of extrude and revolve. To express extrude feature and revolve feature in an unified form, their XML form of expression is defined in this paper, take extrude feature (add material) for example as follows:

$<$ Feature $>$

$<$ Feature ID $>\# \#<$ Feature ID $>$

$<$ Feature_Type $>$ Extrude $<$ /Feature_Type $>$

$<$ Feature_Form $>$ Add_Material $</$ Feature_Form $>$

$<$ Sketch $>$

$<$ Sketch_Plane/ $>$

$<$ Sketch Entity/>

$</$ Sketch $>$

$<$ Sketch Constrains/>

$<$ Extrude_Direction $>0,1,0</$ Extrude_Direction $>$

$<$ End_Condition/ $>$

$</$ Feature $>$

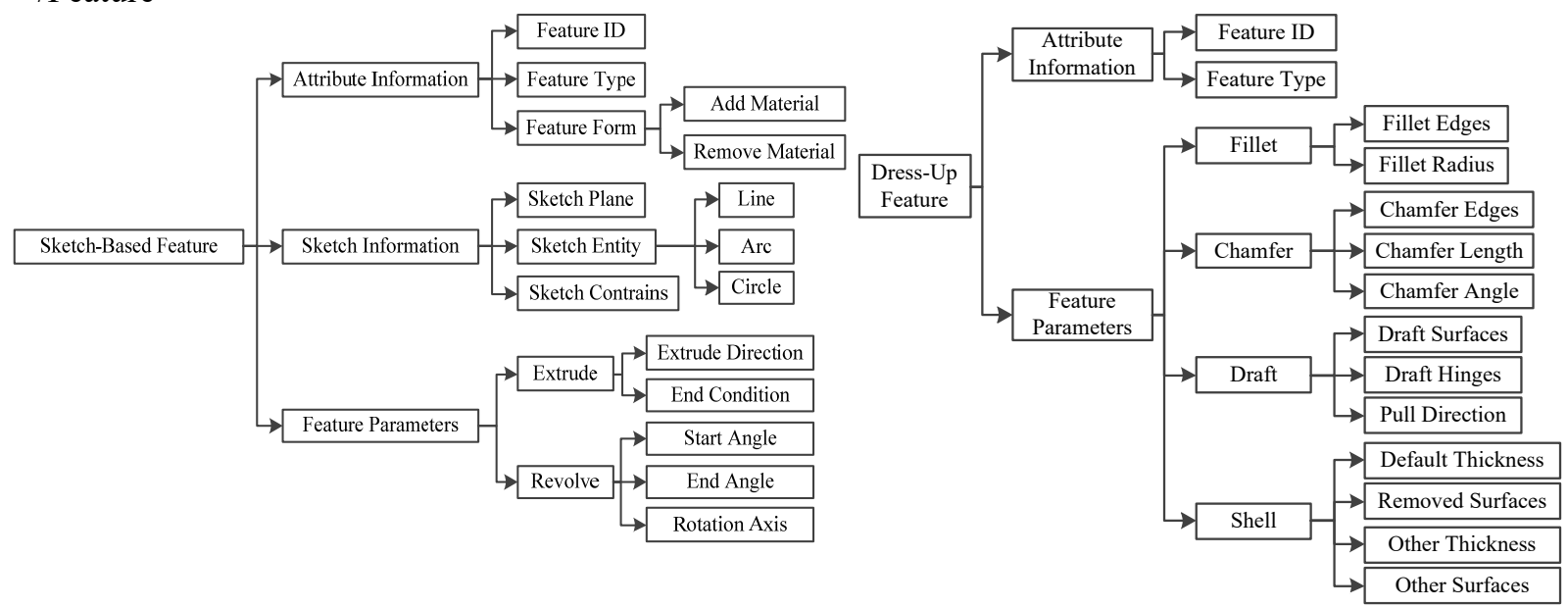

Fig.3 Information of sketch-based feature

Fig.4 Information of dress-up features

Among them, the sketch plane (Sketch_Plane) is represented by the coordinate origin, horizontal axis vector and vertical axis vector in the plane; Sketch entity (Sketch_Entity) includes line, arc and circle; Sketch constraints (Sketch_Constraints) is the constrains among sketch entities, including parallel, perpendicular, symmetry, etc; End condition (End_Condition) includes three forms: given depth and direction, until the next and until the last.

Hole feature is different from extrude feature and revolve feature, the information contained in hole includes location of hole center, hole direction, hole depth and hole dirmeter.

(2) Dress-up feature

Dress-up feature, including fillet, chamfer, draft, shell and etc, is created on other features, playing a modification role. Such feature can't be builded alone, can only be established on the basis of other features. Fig. 4 shows the information contained in dress-up feature. For draft, the XML expression of dress-up feature is as follows:

$<$ Feature $>$

$<$ Feature ID $>\# \#</$ Feature ID $>$

$<$ Feature_Type $>$ Draft $</$ Feature_Type $>$

$<$ Draft_Surfaces/>

$<$ Draft Hinges/ $>$

$<$ End_Condition/>

$<$ Feature $>$

(3) Transformation feature

Transformation feature, including rectangular pattern, circular pattern and etc, is to create a copy of one or more features, by transform operation of existing features. The information contained in transformation feature is as shown in Fig.5. 


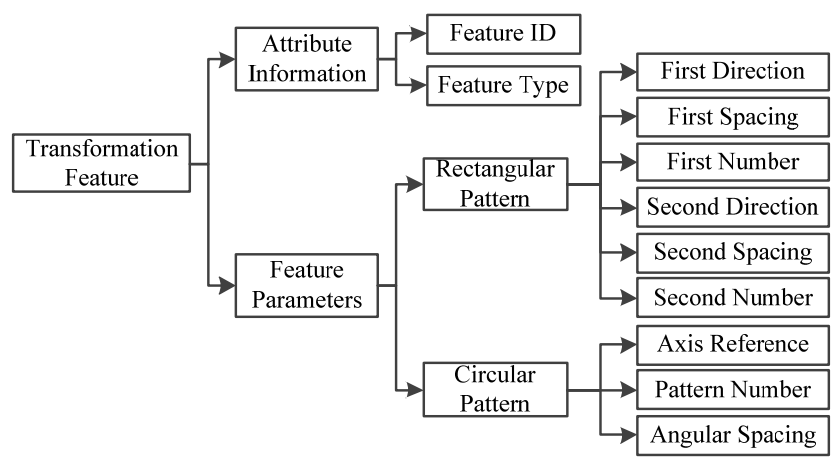

Fig.5 Information of transformation features

Take rectangular pattern as example, the XML expression of transformation feature is as follows: $<$ Feature $>$

$<$ Feature_ID $>\# \#</$ Feature_ID $>$

$<$ Feature Type $>$ Rec Pattern $<$ /Feature Type $>$

$<$ First Direction $>1, \overline{0,0}</$ First Direction $>$

$<$ First_Number $>2<$ First_Number $>$

$<$ First Spacing $>10.0<$ First Spacing $>$

$<$ Second_Direction $>0,1,0</$ Second_Direction $>$

$<$ Second_Number $>2</$ Second_Number $>$

$<$ Second_Spacing $>10.0</$ Second_Spacing $>$

$<$ Feature $>$

\subsubsection{Engineering Notes Information Representation}

As for engineering notes of MBD dataset, according to MBD dataset structure, they are divided into two classes: non feature-associated class and feature-associated class.

(1) Non-feature-associated class

Non-feature-associated class, including standard notes, part notes, material description, is not associated with specific feature, but belongs to the whole part. Take Part Notes as example.

Part notes mainly describe product definition information which is necessary during technology planning, including heat treatment, part final treatment and part mark etc. The general format of part notes is as shown in table 1 .

Table 1 Engineering note item

\begin{tabular}{cccc}
\hline $\begin{array}{c}\text { Parameter } \\
\text { Name }\end{array}$ & Code & Note Content & URL \\
\hline SN00012 & SN00012 & $\begin{array}{c}\text { products need shot } \\
\text { peening and } \\
\text { provided a test } \\
\text { report }\end{array}$ & $\begin{array}{c}\text { http:/standards.we } \\
\text { b.test.com/hlgw.cgi } \\
\text { ?app=BAC\&spec= } \\
\text { BAC2912 }\end{array}$ \\
\hline
\end{tabular}

In table 1, parameter name is defined by code; Code is the unique identification number of the engineering note item; Note content is the specific content that the engineering note item describes; URL is the external link address of the engineering note item.

(2) Feature-associated class

Feature-associated class, including annotation notes, is associated with one or more features. The general format of annotation notes is similar to the part notes. The difference is that the parameter name of annotation notes is defined by the identification number of its parent feature, not named after code.

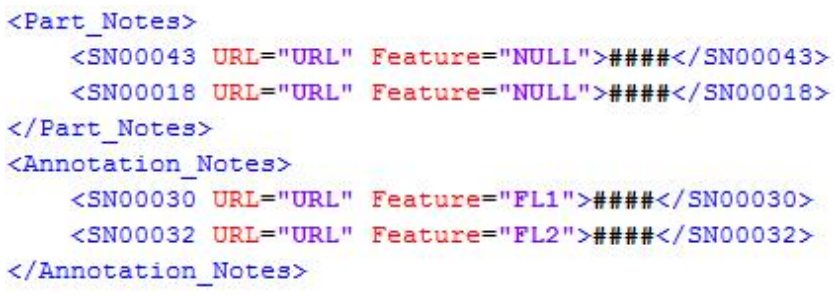

Fig.6 XML expression form of engineering notes 
To express non feature-associated class and feature-associated class with an unified format, the XML representation of engineering note information is defined, as shown in Fig. 6.

\subsection{Information Exchange of heterogeneous MBD model}

To exchange MBD model among heterogeneous CAD systems, use XML file as medium on the basis of the unified XML representation of heterogeneous MBD model. It needs exchange not only geometric feature information, but also non-geometrical information associated with geometry information.

\subsubsection{Overall Architecture}

Fig.7 shows the overall architecture in this paper. It is based on the feature level and mainly includes two parts: (1) Information extraction of the MBD model in the source CAD system; (2) MBD model reconstruction in the target CAD system.

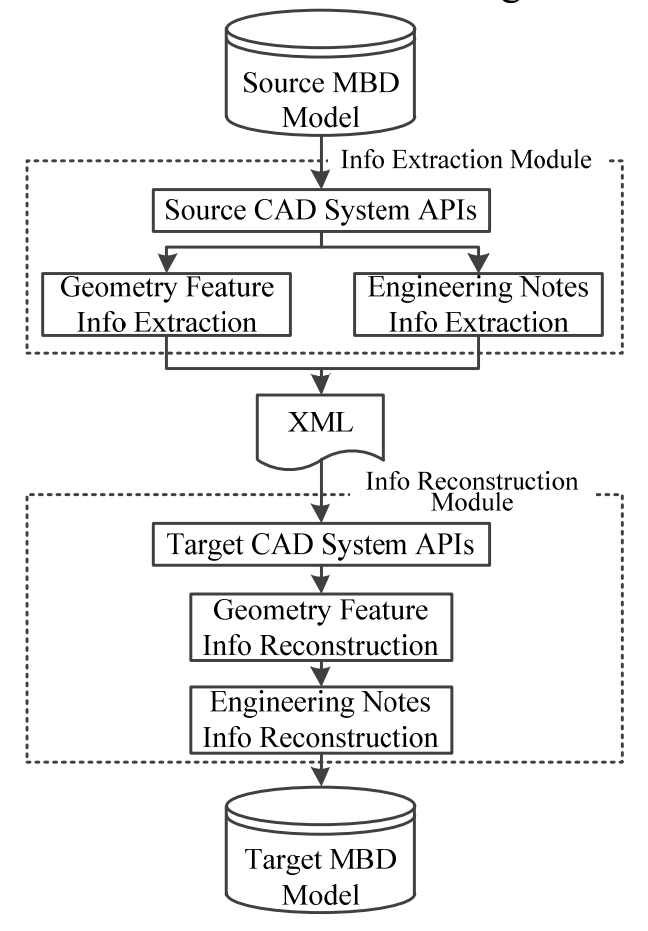

Fig.7 Overall architecture of heterogeneous MBD model exchange

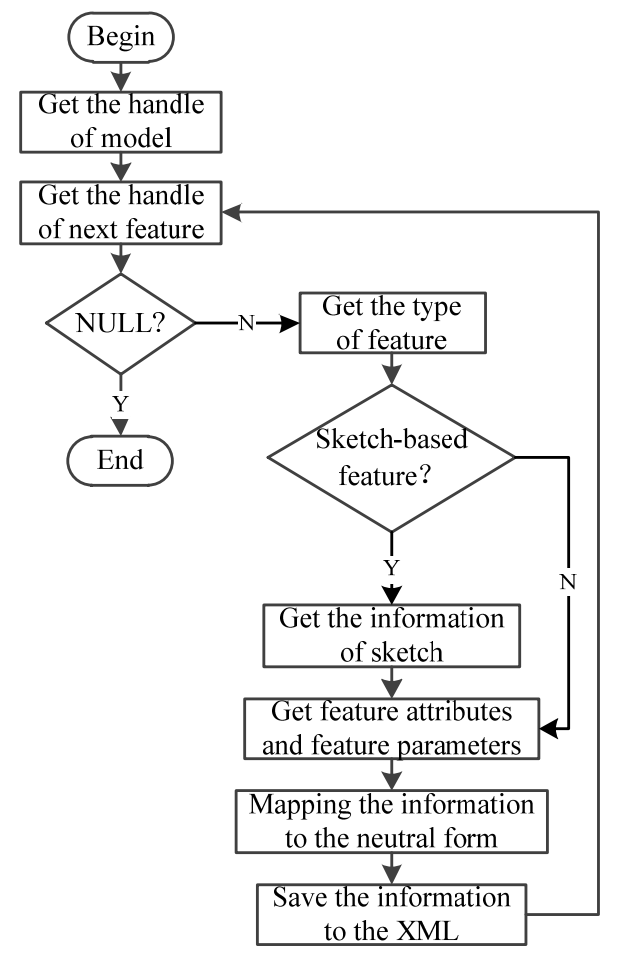

Fig. 8 Extraction of the geometry feature information

In the process of exchanging information of MBD model, there are two types of information need to be exchanged: geometric feature information and engineering notes information. They are not independent, but interrelated with each other.

\subsubsection{Exchange of Geometric Feature Information}

The exchange of geometric feature information includes two steps: geometric feature information extraction and geometric feature information reconstruction. Feature-based CAD system will generate a feature tree which records the geometric feature information in the process of modeling. The process of geometry feature information extraction is show in Fig.8. In source CAD system, extract geometric feature information one by one according to the feature tree. In target CAD system, by parsing the XML file, obtain the complete information needed by geometric feature reconstruction, then call the internal API to reconstruct the features one by one until the model is completely recreated.

In the process of geometric feature information exchange, there are three key problems need to be solved: feature mapping, coordinate system conversion and referenced topology element transformation.

(1) Feature mapping

Table 2 shows the common features and the corresponding features of Pro/E, CATIA, and UG, describes the mapping relation of the common feature type. 
Due to the same type of feature generally have many generated methods, so the different generated methods mapping of feature are also needed to be provided. For extrude feature, neutral form has defined three methods, while Pro/E has six methods and CATIA has five methods, their mapping relation is as shown in table 3 .

Table 2 Common feature type mapping table

\begin{tabular}{|c|c|c|c|c|}
\hline & Common features & Pro/E & CATIA & UG \\
\hline \multirow{4}{*}{$\begin{array}{c}\text { Sketch-Based } \\
\text { Features }\end{array}$} & Extrude(Add /Remove) & Protrusion & Pad & \multirow{2}{*}{$\begin{array}{c}\text { Extrude } \\
\text { Body }\end{array}$} \\
\cline { 2 - 4 } & Revolve(Add/Remove) & Revolve & Shaft & $\begin{array}{c}\text { Revolved } \\
\text { Body }\end{array}$ \\
\cline { 2 - 4 } & Hole & Hole & Hole & Hole \\
\hline \multirow{4}{*}{$\begin{array}{c}\text { Dress-Up } \\
\text { Features }\end{array}$} & Fillet & Round & Edge Fillet & Edge Blend \\
\cline { 2 - 5 } & Chamfer & Chamfer & Chamfer & $\begin{array}{c}\text { Edge } \\
\text { Chamfer }\end{array}$ \\
\cline { 2 - 4 } & Draft & Draft & Draft Angle & Draft \\
\cline { 2 - 4 } & Shell & Shell & Shell & Hollow \\
\hline \multirow{2}{*}{$\begin{array}{c}\text { Transformation } \\
\text { Features }\end{array}$} & Rectangular Pattern & Pattern & $\begin{array}{c}\text { Rectangular } \\
\text { Pattern }\end{array}$ & $\begin{array}{c}\text { Rectangular } \\
\text { Array }\end{array}$ \\
\cline { 2 - 3 } & Circular Pattern & Circular & Circle Array \\
\hline
\end{tabular}

Table 3 Generated method mapping for extrude feature

\begin{tabular}{|c|c|c|}
\hline Pro/E & Neutral Format & CATIA \\
\cline { 1 - 1 } Extrude from sketch plane by a specified depth value & & Dimension \\
\cline { 1 - 1 } Extrude to intersect with selected surface & Provide a depth \\
value and direction & Up to a plane \\
\cline { 1 - 1 } $\begin{array}{c}\text { Extrude on botected point, curve, plane or surface } \\
\text { specified depth value in each direction }\end{array}$ & Up to a surface \\
\hline Extrude up to next surface & Up to next surface & Up to next \\
\hline Extrude to intersect with all surface & Up to last surface & Up to last \\
\hline
\end{tabular}

Table 3 shows that the generated method of extrude to specific object (point, line, face, etc.) can be convert into the generated method of provided a depth value and direction, through calculating the distance and direction from the sketch plane to the specific object.

(2) Coordinate system conversion

The key of the sketch-based feature exchange is to exchange the sketch information. However, the sketch entities' coordinates obtained from source CAD system is based on a $2 \mathrm{D}$ coordinate system which is created arbitrarily by the system, when the sketch information is reconstructed in target CAD system, another 2D coordinate system will be randomly created, then these two coordinate systems may be different, and will lead to failure of feature exchange. To solve this problem, it needs to ensure that the two coordinate systems are the same. Generally, CAD system provides a transformation matrix that goes from $2 \mathrm{D}$ coordinates within the sketch to $3 \mathrm{D}$ coordinates of the owning part, and the 3D coordinates are all the same in heterogeneous CAD systems. Based on this principle, firstly, get the $2 \mathrm{D}$ coordinate system origin, horizontal axis vector and vertical axis vector of the sketch in source CAD system; Secondly, get the transformation matrix of the sketch, and use it to convert $2 \mathrm{D}$ coordinates within the sketch into $3 \mathrm{D}$ coordinates on the basis of the absolute coordinate system; Finally, create the $2 \mathrm{D}$ coordinate system according to the $3 \mathrm{D}$ coordinates in target $\mathrm{CAD}$ system. In this way, the $2 \mathrm{D}$ coordinate systems are ensured the same in heterogeneous CAD systems.

(3) Referenced topology element transformation

In the process of the geometric feature exchange, it's usually needed to transform referenced topology element, such as draft faces of draft feature, fillet edges of fillet feature, and etc. During the reconstruction of geometric feature, in order to accurately reference the topology element, it's 
necessary to give the topology element a correct identification. According to the common situation of the modeling procedure, the identifying solution of curve and face is given in this paper.

The identification of curve includes three parts: curve type, identification of parent feature and parameters. Curve type includes: line, arc and circle, parameters are related to the curve type, as shown in Fig.9.

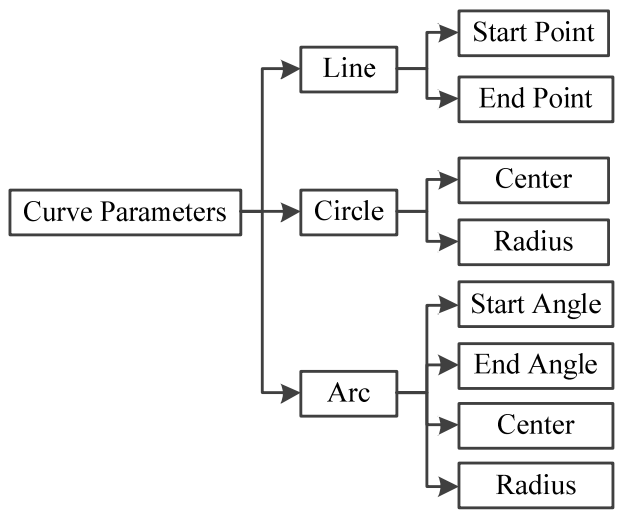

Fig.9 Parameters of curve

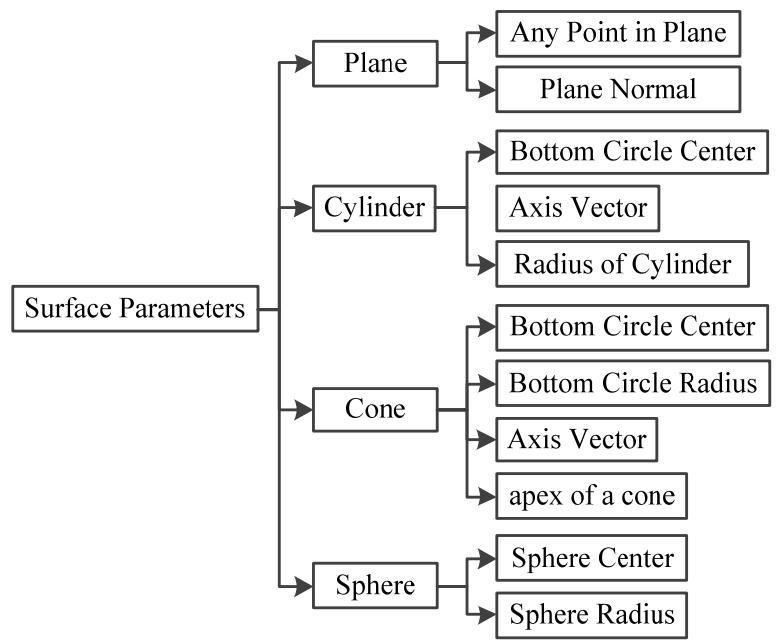

Fig.10 Parameters of surface

\subsubsection{Exchange of Engineering Notes Information}

The exchange of engineering notes information also includes two steps: information extraction and information reconstruction. For non-feature-associated class, firstly, extract the code, note content and URL of engineering notes in source CAD system and write them into a XML file in the XML representation form. Then, get the engineering notes information from the XML file and use the code to define the parameter name of engineering note item. After the data is prepared, write it into the recreated model through the internal API in target CAD system. For feature-associated class, it needs to extract the identification of the feature that the engineering note item belongs to besides the code, note content and URL of the engineering note item in the source CAD system. Then write them into a XML file in the XML representation form. After that, in target CAD system, get the engineering notes information from the XML file and name the parameter name after the identification that the engineering note item belongs to. After the data is prepared, lock the corresponding feature of the recreated model according to the identification of the feature that the engineering note item belongs to and create 3D annotation on the corresponding feature. The content of the 3D annotation is defined by the identification of the feature. As shown in Fig.11, lastly create annotation notes to indicate local specific requirements of the feature associated with the 3D annotation.

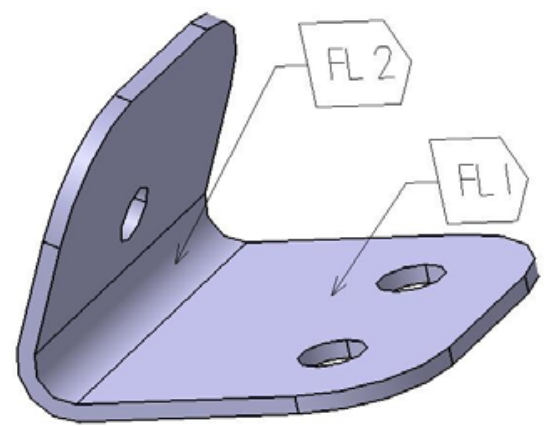

Fig.11 3D annotation associated with feature 


\section{Application and Verification}

According to the method proposed in this paper, take a structural model of a certain type of aircraft as example. We realize the MBD model exchange between heterogeneous CAD systems through secondary development in Pro/E and CATIA.

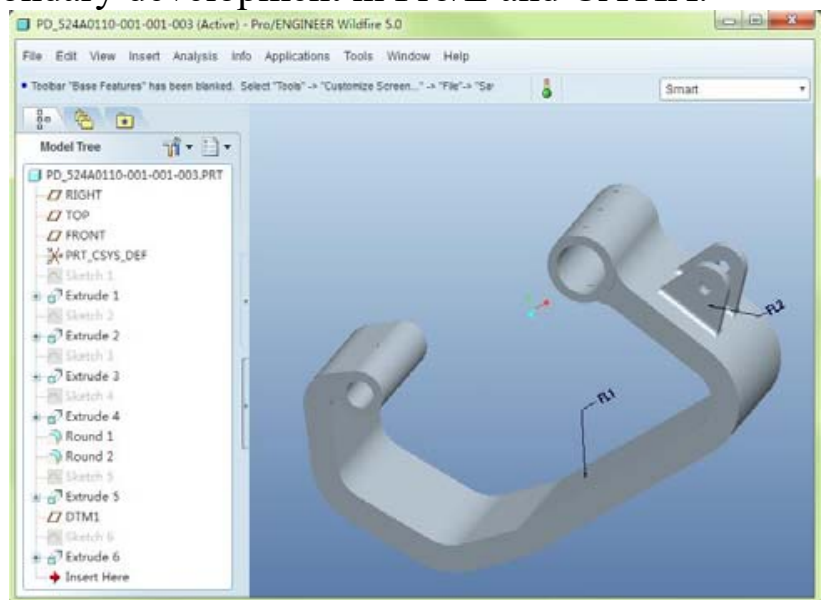

(a) Geometry feature information in Pro/E

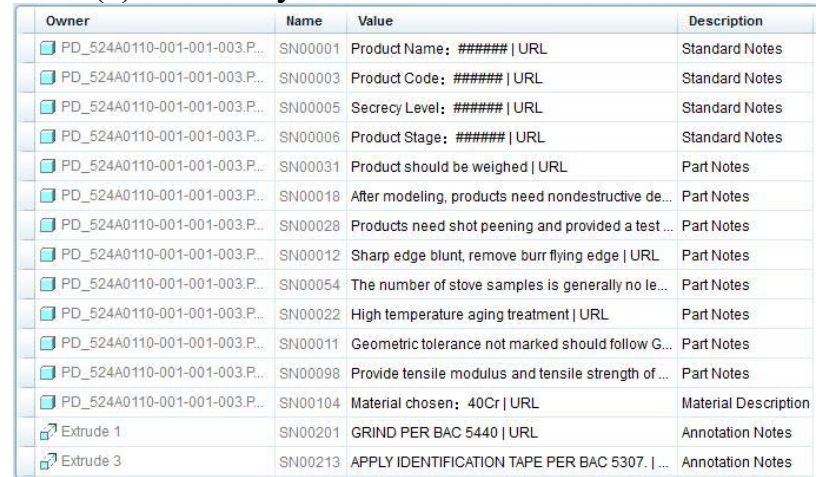

(c) Engineering notes in Pro/E

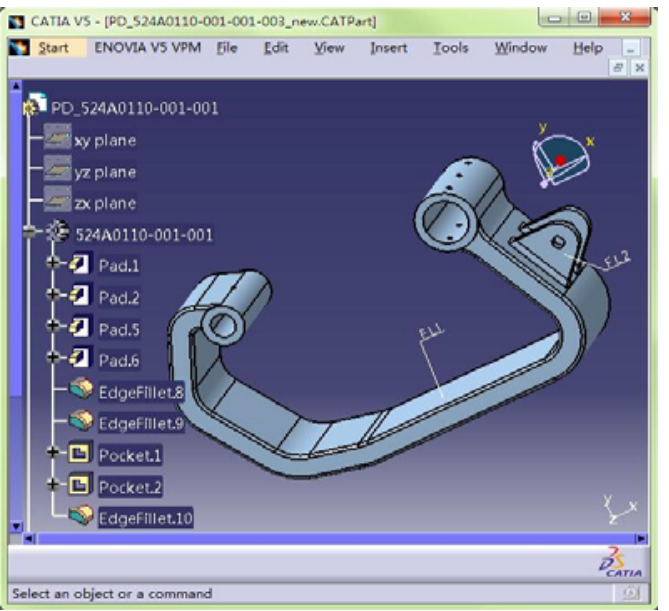

(b) Geometry feature information in CATIA

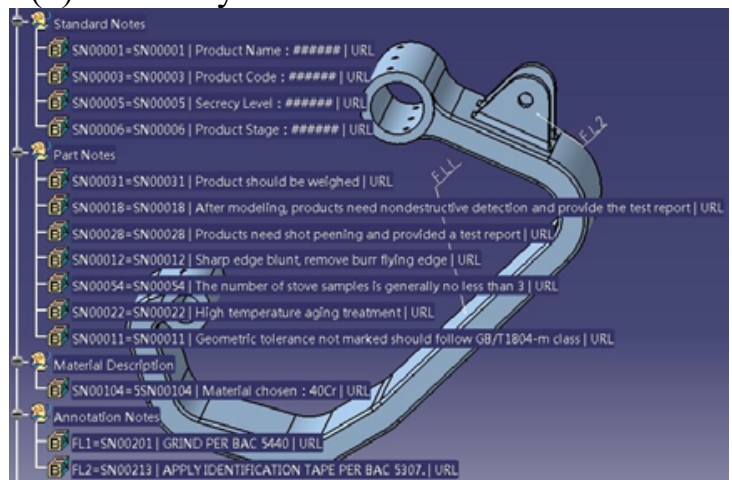

(d) Engineering notes in CATIA

Fig.12 A case-study between two mainstream CAD systems

Fig. 12 shows an example that exchange MBD model from Pro/E to CATIA. Compare Fig.12(a) and Fig.12(b), it can be seen that the geometric feature information of the model isn't lost, the design intent is kept; Compare Fig.12(c) and Fig.12(d), it can be seen that the engineering notes information is successfully exchanged, the identifications of the parent features that the annotation notes belong to are FL1 and FL2, the 3D annotations and the annotation notes completely represent the correlation between Feature-associated engineering notes and geometric features.

\section{Summary}

This paper is aimed at MBD model, considering the geometrical information and non-geometrical information, the MBD model information is divided into geometric feature information and engineering notes information, according to the MBD dataset normative definition requirements. We use XML to represent the MBD model information and the correlation between geometric feature information and engineering notes information. By improving feature-based data exchange method, MBD model exchange between heterogeneous CAD systems is realized. Firstly, extract the MBD model information and write it into a XML file. Then, reconstruct the information on the basis of the XML file and keep the correlation between geometric feature information and engineering notes information. At last, use CAD secondary development technology to exchange a structural model of a certain type of aircraft between Pro/E and CATIA, and validity of the method is verified. This paper is aimed at solid feature. However, complex surface feature is generally composed by many child features, and some of the child features are uniquely owned by one CAD system, so their feature 
parameters have a great difference between CAD systems. In this reason, the exchange of complex surface feature is difficult, and more research is needed.

\section{Acknowledgments}

This paper has been sponsored by National 863 Project (2009AA043302). The corresponding author of this paper is Yu Yong, and her email address is yuyong@buaa.edu.cn.

\section{References}

[1] Gao Shuming, He Fazhi. Survey of distributed and collaborative design[J]. Journal of Computer-Aided Design \& Computer Graphics, 2004, 16(2): 149-157.

[2] Alemanni M, Destefanis F, Vezzetti E. Model-based definition design in the product lifecycle management scenario[J]. The International Journal of Advanced Manufacturing Technology. 2011, 52(1-4): 1-14.

[3] Tessier S, Wang Y. Ontology-based feature mapping and verification between CAD systems[J]. Advanced Engineering Informatics, 2013, 27(1):76-92.

[4] Hoffmann C M, Juan R. Erep, an editable, high-level representation for geometric design and analysis[C]. Geometric Modeling for Product Realization, Selected and Expanded Papers From the Ifip Tc5/wg5.2 Working Conference on Geometric Modeling, Rensselaerville, Ny, Usa, 27 September - 1 October. 1992:129-164.

[5] Choi G H, Mun D, Han S. Exchange of CAD Part Models Based on the Macro-Parametric Approac[C]. INTERNATIONAL JOURNAL OF CAD/CAM. 2002:13--21.

[6] Mun D, Han S, Kim J, et al. A set of standard modeling commands for the history-based parametric approach[J]. Computer-Aided Design, 2003, 35(13):1171-1179.

[7] Rappoport A. An architecture for universal CAD data exchange[C]. Eighth ACM Symposium on Solid Modeling and Applications 2003, Seattle, Washington, USA, June. 2003:266-269.

[8] Rappoport A, Spitz S, Etzion M. Two-Dimensional Selections for Feature-Based Data Exchange[M]. Geometric Modeling and Processing - GMP 2006. Springer Berlin Heidelberg, 2006:325-342.

[9] Spitz S, Rappoport A. Integrated feature-based and geometric CAD data exchange[J]. Proc.acm Symp.solid Modelingand Applic, 2004:183-190.

[10] Li X, Cai X, He F, et al. Retrieval and reconstruction of heterogeneous feature data for collaborative design[C]. International Conference on Computer Supported Cooperative Work in Design. 2010:553-558.

[11] Li X, He F, Cai X, et al. CAD data exchange based on the recovery of feature modelling procedure[J]. International Journal of Computer Integrated Manufacturing, 2011, 25(10):1-14.

[12]Fan Yuqing. Model Based Definition Technology and Its Practices[J]. Aeronautical Manufacturing Technology, 2012(06): 42-47.

[13] Lu Hu, Han Shuang, Fan Yuqing. Model Based Digital Definition Technology[J]. Aeronautical Manufacturing Technology, 2008(3):78-81. 